\title{
TRASPLANTE ALOGÉNICO DE CÉLULAS PROGENITORAS HEMATOPOYÉTICAS DE SANGRE PERIFÉRICA CON ACONDICIONAMIENTO DE BAJA INTENSIDAD COMO TRATAMIENTO DEL CARCINOMA DE CÉLULAS CLARAS METASTÁSICO. RESULTADOS PRELIMINARES CON DOS AÑOS DE SEGUIMIENTO MÍNIMO.
}

José Pablo Maroto, Rodrigo Martino', Humberto Villavicencio², José Rosales² y Jordi Sierra?.

Servicio de Oncología Médica, Servicio de Hematologíal y Servicio de Urología².

Hospital de Sant Pau. Barcelona. España.

Resumen.- OBJETIVO: Evaluar la toxicidad y resultados de transplante alogénico de células progenitoras hematopoyéticas con intensidad reducida (mini-alo TMO) en carcinoma renal metastásico refractario al menos a 1 línea de tratamiento sistémico.

MÉTODOS: De 30 pacientes remitidos al Hospital de Sant Pau para inmunoterapia entre l/2001 y l/2003, 6 pacientes fueron finalmente sometidos a mini-alo TMO en régimen de ensayo. Acondicionamiento: Fludarabina: $30 \mathrm{mg} / \mathrm{m} 2$ en los días $-9,-8,-7,-6$ y -5 en infusión IV de 30 minutos. Busulfán: $1 \mathrm{mg} / \mathrm{kg}$ (peso real) cada 6 horas v. o. los días $-6,-5$ y -4 ( 4 dosis al día -6 y -5 y sólo 2 dosis el -4, 10 dosis en total).

RESULTADOS: Dos pacientes alcanzaron una RP, un paciente una EE y dos pacientes progresaron $a+30$ y +60 . Por tanto, 2 de los 3 pacientes que sobrevivieron más de 6 meses alcanzaron una RP. Un paciente falleció al día +30 por enfermedad injerto contra huésped aguda hepática. Se demostró un quimerismo completo en todos los pacientes.

CONCLUSIÓN: El mini-alo TMO es factible en pacientes con carcinoma renal metastásico y proporciona respuestas objetivas, si bien su eficacia debería confirmarse en ensayos fase III.

José Pablo Maroto Rey Servicio de Oncología Médica C/Padre Ma Claret 167 Hospital de Sant Pau 08025 Barcelona. (España) baldomir@yahoo.com Trabajo recibido: 7 de julio 2005
Palabras clave: Cáncer de riñón. Trasplante alogénico de células progenitoras.

Summary.- OBJECTIVES: To evaluate the toxicity and outcomes of the allogeneic transplantation of peripheral blood hematopoietic stem-cells with low intensity conditioning Imini-alo Bone Marrow transplantationBMT) in metastatic renal cell carcinoma refractory to at least one line of systemic treatment.

METHODS: From 30 patients submitted to the Sant Pau's Hospital for immunotherapy between 1/2001 and 1/2003, six patients finally underwent mini-alo BMT within a clinical trial. Conditioning: Fludarabine: 30 minute $\mathrm{IV}$ infusion of $13 \mathrm{mg} / \mathrm{m} 2$ on days - 9, - 8, - 7, - 6 and - 5. Busulfan: $1 \mathrm{mg} / \mathrm{kg}$ (real weight) q 6 hours, $P O$ on days - $6,-5$, and -414 doses per day on days -6 and -5 , and 2 doses only on day - 4, total number of doses 10).

RESULTS: Two patients achieved partial response, one patient stabilized disease, and two patients had progression on days +30 and +60 . Therefore, $2 / 3$ patients surviving more than six months achieved partial response. One patient died on day +30 after acute hepatic graft-versus-host disease. Complete chimerism was demonstrated in all patients.

CONCLUSIONS: The mimni-alo BMT is feasible in patients with metastatic renal cell carcinoma and provides objective responses, although its efficacy should be confirmed by phase III clinical trials.

Keywords: Kidney cancer. Peripheral. Allogeneic cell transplant. 


\section{INTRODUCCIÓN}

Un tercio de los pacientes con carcinoma renal (CCR) tienen metástasis en el momento del diagnóstico. La mediana de supervivencia de los pacientes con metástasis es de 10 meses y menos de un $2 \%$ son largos supervivientes, porcentajes que no han mejorado significativamente en los últimos 25 años. En el CCR metastásico los tratamientos oncológicos clásicos, hormonoterapia y quimioterapia, son poco eficaces, y la inmunoterapia continúa como estándar (1).

La literatura médica recoge comunicaciones anecdóticas de regresión de metástasis de tumores sólidos por el efecto inmunomodulador de un injerto en el contexto de transplante alogénico de médula ósea realizado por otra causa. En el año 2000 Childs y cols. publicaron por primera vez una serie homogénea de tratamiento con mini-alo TMO del CCR metastásico (2), en la que se describían hasta un $40 \%$ de respuestas completas y un $60 \%$ de respuestas globales, muy superior a lo publicado con cualquier otro tratamiento en esta enfermedad.

Con menor toxicidad que el transplante de médula ósea alogénico clásico, la técnica de minialo TMO consiste en crear un corto período "ventana" de inmunosupresión que permite el implante con un acondicionamiento no mieloablativo, a diferencia del transplante procedente de médula ósea (3). El objetivo es la utilización del injerto como inmunoterapia activa. Los primeros resultados con esta técnica de tratamiento en CCR metastásico se están ahora comunicando (4-7).

Para valorar la factibilidad y eficacia iniciamos en 2001 un ensayo fase II de minialo TMO como inmunoterapia para CCR metastásico. Presentamos los resultados de los pacientes con al menos dos años de seguimiento.

\section{PACIENTES Y MÉTODOS}

\section{Criterios de Inclusión.}

Treinta pacientes con CCR metastásico fueron remitidos al Servicio de Oncología entre I/2001 y l/2003, lo que constituye ya un grupo muy seleccionado para tratamiento con inmunoterapia (en nuestro Hospital IL-2 $18 \times 10^{6} \mathrm{UI} /$ día/5 días por semana s.c. e Interferón alfa $2 a 6 \times 10^{6} \mathrm{UI} / 3$ días por semana s.c). A la progresión, 13 pacientes cumplían los criterios de inclusión en el ensayo de minialo TMO que eran:

- Consentimiento informado.

- Edad <60 años.
- Nefrectomía previa.

- No metástasis cerebrales.

- Buena función de órganos.

- Buen estado general (Indice escala Karnofsky $>70 \%$ ).

Los pacientes se excluyeron si tenían infección activa o diabetes. Se consideró necesaria la nefrectomía previa.

De estos 13 pacientes, 7 tenían un donante relacionado HLA idéntico o un mismatch en un antígeno. Uno de ellos rechazó continuar con el procedimiento y finalmente fueron 6 los pacientes transplantados.

\section{Recolección de células progenitoras del donante.}

Se administró G-CSF 5 ug $/ \mathrm{kg}$ s.c. dos veces al día por 3 días antes de la recolección de las células progenitoras y hasta el final de la recolección. Las leucoaféresis se continuaron hasta un mínimo de $4 \times 10^{6}$ de células CD34+/ $\mathrm{Kg}$.

\section{Acondicionamiento y procedimiento de transplante.}

- Régimen de acondicionamiento: consistió de Fludarabina y Busulfán, según la siguiente dosis y esquema: Fludarabina: $30 \mathrm{mg} / \mathrm{m}^{2}$ en los días $-9,-8,-7,-6$ y -5 en infusión IV de 30 minutos. Busulfán: $1 \mathrm{mg} / \mathrm{kg}$ (peso real) cada 6 horas v.o. los días $-6,-5$ y -4 (4 dosis al día -6 y -5 y sólo 2 dosis el $-4,10$ dosis en total).

- Profilaxis EICH: Se administró $1 \mathrm{mg} / \mathrm{kg} /$ día de ciclosporina A (CSA) en infusión IV continua o cada 12 $\mathrm{h}$ desde el día -7 y hasta el día -2. El día -1 se cambia la dosis a $2 \mathrm{mg} / \mathrm{kg} /$ día $(1 \mathrm{mg} / \mathrm{kg} / 12 \mathrm{~h}$ en infusión de 1-2 h) hasta el paso a v.o., en que se dobla la dosis necesaria de la i.v. para mantener un nivel en sangre de 150-300 $\mathrm{ng} / \mathrm{ml}$. En caso de no aparecer EICH aguda > grado I, a partir del día +90 se disminuyó la dosis a ritmo de un $10 \%$ semanal o un $20 \%$ cada 2 semanas hasta su retirada alrededor del día +150 . Se administraron $10 \mathrm{mg} / \mathrm{m} 2$ de Metotrexate i.v. los días $+1,+3$ y +6 .

\section{INJERTO Y MEDIDAS DE SOPORTE}

El injerto se definió como el primero de 2 días consecutivos con un número de neutrófilos absoluto $\geq 0.5 \times 109 / \mathrm{L}$. Se transfundieron concentrados de plaquetas y hematíes para mantener un nivel de plaquetas mayor de 10×109/L y de hemoglobina por encima de $8 \mathrm{~g} / \mathrm{dL}$. Todos los productos sanguíneos se irradiaron y filtraron antes de la transfusión. 
Los pacientes recibieron profilaxis antibacteriana y antifúngica que consistió de ciprofloxacino oral $500 \mathrm{mg} / 12 \mathrm{~h}$ y fluconazol $200 \mathrm{mg} / 24 \mathrm{~h}$. La profilaxis antivírica consistió de aciclovir $800 \mathrm{mg} / 12$ h v.o. La profilaxis de la neumonía por P. Carinii se inicia antes del trasplante con una dosis de pentamidina inhalada en el día -7. Prosigue con cotrimoxazol 1 comprimido/ $12 \mathrm{~h}$ martes y jueves en cuanto se produce la recuperación hematológica y hasta el día +180 . En caso de toxicidad hematológica o intolerancia a este fármaco se empleó pentamidina inhalada, $300 \mathrm{mg}$ cada 4 semanas.

\section{RESULTADOS}

Entre I/2001 y l/2003, 6 pacientes recibieron un transplante de células progenitoras hematopoyéticas de sangre periférica con acondicionamiento de baja intensidad. Cinco recibieron un transplante de un donante HLA idéntico y 1 de un donante relacionado con un micro mismatch en un focus HLA. El rango de edad fue de 29 a 54 años (mediana 36).

\section{TRANSPLANTE E INJERTO}

El procedimiento se llevó a cabo en régimen hospitalario. La toxicidad en forma de $\mathrm{EICH}$ se resume en la Tabla I.

La cuenta de neutrófilos se redujo a menos de $100 / \mathrm{mm}^{3}$ en todos los pacientes y permaneció por debajo de $500 / \mathrm{mm}^{3}$ una mediana de 8 días (rango 7-13). Todos los pacientes presentaron fiebre. La mediana de días hasta la recuperación de plaquetas $\left(>50.000 / \mathrm{mm}^{3}\right)$ fue de 8 días $(6-21)$.

Todos los pacientes se recuperaron con una quimera mixta.

\section{ENFERMEDAD INJERTO CONTRA HUÉSPED}

Como se mencionó, un paciente falleció por $\mathrm{EICH}$ hepática aguda, ningún otro caso presentó $\mathrm{EICH}$ agudo. Un segundo paciente presentó $\mathrm{EICH}$ crónico hepático y pulmonar grado IV complicado con aspergillosis pulmonar invasiva que finalmente fue la causa

TABLA I. EVOLUCIÓN DE LOS 6 PACIENTES TRANSPLANTADOS.

\begin{tabular}{|c|c|c|c|c|c|c|c|}
\hline Edad & Metástasis & ILE (m) & $\begin{array}{c}\text { Tratamiento } \\
\text { previo }\end{array}$ & ILP (m) & $\begin{array}{l}\text { Toxicidades etra- } \\
\text { hematológicas } \\
\text { graves }\end{array}$ & $\mathrm{EICH}$ & \begin{tabular}{|l} 
Máximas \\
Respuestas \\
Tumorales
\end{tabular} \\
\hline 52 & Pulmón & 24 & QT/IL-2 & +1 & $\begin{array}{l}\text { Insuficiencia } \\
\text { renal aguda }\end{array}$ & No & Prog \\
\hline 48 & $\begin{array}{c}\text { Hueso } \\
\text { Supraclavic }\end{array}$ & 24 & $\mathrm{IL}-2 / \mathrm{Ifn}$ & +1 & Gastrointestinal & Aguda, grado IV (mortal) & NE \\
\hline 32 & $\begin{array}{l}\text { Pulmón } \\
\text { Hueso }\end{array}$ & 24 & QT/IL-2/Ifn & +2 & No & No & Prog \\
\hline 33 & Pulmón & 9 & IL-2/Ifn & +13 & No & $\begin{array}{c}\text { Crónica leve, tras } \\
\text { infusión de linfocitos del } \\
\text { donante + IFN }\end{array}$ & $\mathrm{RP}>50 \%$ \\
\hline 35 & $\begin{array}{l}\text { Hueso } \\
\text { Ganglionar }\end{array}$ & 0 & IL-2/Ifn & +6 & No & $\begin{array}{c}\text { Aguda moderada (grado } \\
\text { 3), crónica extensa }\end{array}$ & $\operatorname{RP}(50 \%)$ \\
\hline 29 & Ganglionar & 0 & IL-2/Ifn & +6 & No & & $\mathrm{EE}$ \\
\hline
\end{tabular}

*Todos los intervalos calculados desde la fecha de transplante. ILP: Intervalo libre de progresión. MCE: Muerto con enfermedad. VCE: Vivo con enfermedad. QT: Quimioterapia. EE: Enfermedad estable. RP: Respuesta parcial. Prog: Progresión. 
de muerte a dos años del procedimiento. Dos pacientes presentaron $\mathrm{EICH}$ crónico cutáneo grado II.

\section{EFICACIA}

Las características de los pacientes y su evolución individual se resumen en la Tabla I. De los 6 pacientes, en 2 hubo evidencia de regresión de las lesiones después del transplante, y los 3 pacientes que sobrevivieron por encima de 6 meses mostraron alguna evidencia de eficacia clínica (respuesta parcial o estabilización). Una paciente falleció por progresión cerebral precoz y hemorragia de una metástasis cerebral, y un segundo paciente por progresión pulmonar en el tercer mes de seguimiento con dísnea secundaria. La respuesta fue retrasada en los 2 casos y se describió 6 y 8 meses después del injerto.

El paciente 5 ejemplifica la necesidad de manejo multidisciplinar. Debutó con metástasis pulmonares múltiples que progresaron durante el tratamiento con IL2 e Interferón. Se indicó minialo como rescate, que se realizó sin complicaciones agudas. En el control de los 3 meses existía una progresión de las lesiones pulmonares que habían regresado en número y tamaño en el control a los 6 meses. La respuesta se mantuvo por un año en que se detectó progresión en fosa lumbar que fue rescatada quirúrgicamente. Seis meses más tarde fueron resecados 2 nódulos pulmonares que progresaron radiológicamente, y que en un PET que se realizó en este momento captaban glucosa de manera diferencial con respecto a otros en el parénquima pulmonar adyacente. El paciente se encuentra actualmente vivo con enfermedad a más de 3 años del procedimiento.

\section{TRATAMIENTO A LA PROGRESIÓN}

De los 3 pacientes con respuesta o estabilización, dos se sometieron a infusión de linfocitos activados en el momento de la progresión. En ninguno se objetivó respuesta.

\section{SUPERVIVENCIA GLOBAL Y LIBRE DE ENFERMEDAD}

A 1 de Octubre de 2004, 1 paciente está vivo a 39 meses del transplante. La causa de éxitus fue complicación del transplante en 2 casos, progresión tumoral en 3. Los dos pacientes con RP progresaron a 8 y 13 meses, y el paciente con EE a los 7 meses.

\section{DISCUSIÓN}

El CCR tiene un tratamiento sistémico muy poco eficaz ya que después de treinta años de estudios randomizados sólo han demostrado actividad de manera consistente el interferón alfa y la inter- leukina II $(8,9)$. Pese al bajo índice de respuesta, el carcinoma renal es el tumor de la economía que presenta una tasa más alta de respuesta a maniobras inmunológicas. Así, las altas dosis de IL-2 endovenosa proporcionan un $15 \%$ de respuestas globales y un $6 \%$ de respuestas completas mantenidas en el grupo ya muy seleccionado de pacientes candidatos a este tratamiento. Dosis menores, o la combinación de varios con dosis reducidas disminuye la toxicidad pero también la tasa de respuestas (10).

Romper la tolerancia inmunológica hacia el tumor con los métodos actualmente disponibles es difícil. Desde un punto de vista teórico resulta más fácil substituir un sistema inmunitario incapaz por otro nuevo potencialmente eficaz y no expuesto al efecto de tolerancia del tumor. La tecnología de acondicionamiento de baja intensidad evita grandes dosis de quimio o radioterapia y proporciona una corta ventana que permite el injerto de las células de un donante con menor toxicidad que el clásico transplante alogénico. El régimen de intensidad reducida que utilizamos facilitó un injerto estable en todos nuestros pacientes, si bien con toxicidad no despreciable y el $\mathrm{EICH}$ fue la primera causa de morbi-mortalidad.

De lo publicado hasta la fecha se sugiere que es necesario un intervalo mínimo de 4 - 6 meses para que se desencadene la reacción del injerto contra el tumor, por lo que sólo los pacientes con una esperanza de vida en torno a 1 año serían buenos candidatos (11). En este rango de supervivencia se encuentran los pacientes del grupo de pronóstico intermedio de la clasificación de Motzer (12).

Sólo 20\% de los pacientes remitidos a nuestro centro para inmunoterapia, que constituyen ya de por sí un grupo seleccionado, fueron finalmente transplantados. La toxicidad inherente al procedimiento obliga a una cuidadosa selección de posibles candidatos para optimizar el riesgo/beneficio. Esta selección, en combinación con el manejo óptimo del EICH proporciona, quizá, una alternativa válida para pacientes muy seleccionados de pronóstico intermedio. La identificación de factores moleculares que ayuden a determinar qué pacientes responderán a inmunoterapia maximizará el cociente beneficio-riesgo (13). Así mismo, en los 2 últimos años algunos fármacos de nueva generación se han mostrado prometedores en ensayos fase II, como aniangiogénicos $(14,15)$ o inhibidores de factores de crecimiento o vías de crecimiento celular $(16,17)$. La demostración de su eficacia y posterior inclusión el tratamiento del CCR metastásico proporcionaría un mayor intervalo libre de progresión más que respuestas objetivas (18), intervalo que es importante en el contexto de espera de una respuesta del injerto contra el tumor. 
En la serie presentada, el minialo TMO como inmunoterapia proporcionó respuestas, alguna de ellas duradera, y alteró la evolución natural de una enfermedad sin tratamiento eficaz demostrado en el contexto de fallo a inmunoterapia previa.

\section{BIBLIOGRAFÍA y LECTURAS RECOMENDADAS ( ${ }^{*}$ lectura de interés $y^{* *}$ lectura fundamental)}

1. TABERNERO, J.; SALAZAR, R.; GONZÁLEZ LARRIBA, J.L. y cols.: "The inmunological treatment of renal cancer". Med. Clin., 112: 340, 1999.

**2. CHILDS, R.; CHERNOFF, A.; CONTENTIN, N. y cols.: "Regression of metastatic renal-cell carcinoma after nonmyeloablative allogeneic peripheral-blood stem-cell transplantation". N. Engl. J. Med., 343: 750, 2000.

3. SLAVIN, S.; NAGLER, A.; NAPARSTEK, E. y cols.: "Nonmyeloablative stem cell transplantation and cell therapy as an alternative to conventional bone marrow transplantation with lethal cytoreduction for the treatment of malignant and nonmalignant hematologic diseases". Blood, 91: 756, 1998.

**4. NAKAGAWA, T.; MASAHIRO, K.; AKIKO, H. y cols.: "Allogeneic hematopoietic stem cell transplantation with a reduced-intensity conditioning regimen for treatment of metastatic renal cell carcinoma: single institution experience with a minimum 1-year follow-up". Exp. Hematology, 32: 599, 2004.

5. HU, H.S.; RINI, B.I.; ZIMMERMAN, T.M. y cols.: "Allogeneic stem cell transplantation for metastatic renal cell cancer after non-myeloablative chemotherapy: updated results from the University of Chicago". Proc. Am. Soc. Clin. Oncol., 180, 2002.
6. MAROTO, P.; MARTINO, R.; RAMIREZ, A. y cols.: "Impact of a non-myeloablative allogeneic transplantation programme for metastatic renal cell carcinoma on a medical oncology service". Ann. Oncol., 5: 92, 2002.

7. BREGNI, M.; DODERO, A.; PECCATORI, J. y cols.: "Nonmyeloablative conditioning followed by hematopoietic cell allografting and donor lymphocyte infusions for patients with metastatic renal and breast cancer". Blood, 94: 2409, 2002.

8. FLANIGAN, R.C.; SALMON, S.E.; BLUMENSTEIN, B.A. y cols.: "Nephrectomy followed by interferon alfa- $2 \mathrm{~b}$ compared with interferon alfa-2b alone for metastatic renal-cell cancer". $\mathrm{N}$. Engl. J. Med., 345: 1655, 2001.

9. MOTZER, R.J.: "Renal cell carcinoma: A priority malignancy for development and study of novel therapies". J. Clin. Oncol., 21: 1193, 2003.

*10. YANG, J.C.; SHERRY, R.M.; STEINBERG, S.M. y cols.: "Randomized study of high-dose and lowdose interleukin-2 in patients with metastatic renal cancer". J. Clin. Oncol., 21: 3127, 2003.

*11. PEDRAZZOLI, P.; DA PRADA, G.A.; GIORGIANI, G. y cols.: "Allogeneic blood stem cell transplantation after a reduced-intensity, preparative regimen: a pilot study in patients with refractory malignancies". Cancer, 94: 2409, 2002.

**12. MOTZER, R.J.; BACIK, J.; SCHWARTZ, L.H. y cols.: "Prognostic factors for survival in previously treated patients with metastatic renal cell carcinoma”. J. Clin. Oncol., 22: 454, 2004.

13. ELLERHORST, J.A.; HILDEBRAND, W.H.; CAVETT, J.W. y cols.: "Heterozygosity or homozygosity for 2 HLA Class II Haplotypers predict favorable outcomes for renal cell carcinoma treated with cytokine therapy". J. Urol., 169: 2084, 2003.

14. YANG, J.C.; HAWORTH, L.; SHERRY, R.M. y cols.: "A randomized trial of bevacizumab, an anti-vascular endothelial growth factor antibody for metastatic renal cancer". N. Engl. J. Med., 349: 427, 2003. 